\title{
Miniaturized Passive Two-Path Notch Filter For UWB receiver
}

\author{
R. Lababidi *, A. Mansour, F. Le Roy \\ Lab-STICC- ENSTA Bretagne \\ Brest, France \\ Corresponding author: raafat.lababidi@ensta-bretagne.fr
}

\author{
J. Gaubert, S. Bourdel \\ IM2NP - UMR CNRS 7334, University of Aix-Marseille \\ Campus de Saint-Jérôme, Avenue Escadrille Normandie \\ Niemen-Case 142, F-13397 Marseille Cedex, France
}

\begin{abstract}
In this paper, the RF characteristics of the flexible Kapton-based substrate are investigated by using the microstrip ring resonator in order to characterize the relative permittivity (Er) and loss tangent $(\tan \delta)$ of the substrate at Ultra Wide Band (UWB) range. A compact notch filter using the approach of phase cancellation between two signal paths is also reported here. The passive notch filter is designed onto a Rogers4003-based substrate to test its performance. The integrated silicon chip is then reported on a grounded area of filter and connected to the output of this later by wire-bonding forming an SIP (System In Package) approach. The complete design is fully simulated using HFSS simulation tools, featuring high rejection at the central frequency and very low insertion loss far from the notch frequency.
\end{abstract}

Keywords-Flexible Substrate; Ring Resonator; SIP; Notch Filter; $U W B$ receiver

\section{INTRODUCTION}

Currently, there is significant interest in notch filters for use in advanced communication systems [1], [2]. Indeed, in the presence of out-of-band interferers coming from close jammers, a notch filter can protect the receiver from saturation, and the linearity requirement is significantly improved. In particular, when these jammers operate in frequency bands which are very close from the frequency spectrum of the receiver, highly selective filtering solutions have to be found out in order to ensure their coexistence. Moreover, with the growth of demand for low-cost, flexible, light weight, compact and power-efficient broadband wireless electronics, materials and topologies have to be carefully chosen to meet with the critical challenges imposed by the wireless communication market.

This paper deals with the coexistence of an UWB (Ultra Wide Band) system using the frequency spectrum from 3.1 to $10.6 \mathrm{GHz}$ with IEEE $802.11 \mathrm{~b} / \mathrm{g} / \mathrm{n}$ standards operating at 2.4 $\mathrm{GHz}$. Indeed, transmitted signals according to these standards are very close to the bandwidth of the UWB receiver. Due to this proximity, the UWB receiver can be highly degraded if these interference signals are not properly rejected

From material point of view, flexible substrate is considered one of the best organic substrate candidates for UHF and microwave applications [3]. It is used principally but not only to develop inexpensive RFID tags [4]. Flexible substrate can significantly reduce the weight profile of the device and can provide robustness to impact since flexible things don't break easily when they get hit. Moreover, it is considered among the lowest cost materials used in the industry in terms of mass production. However, electrical properties of this flexible substrate are generally given in the technical data sheet at low frequencies. Thus, to use these materials at RF and microwave frequencies, an RF substrate characterization must be done for an optimal design.

From topological point of view, when compactness, low cost and ease of production are considered in the design of the notch filter, planar structures come forward. However, planar structures have significant circuit losses which reduce the resonator quality factor Q. Consequently, the filter suffers from insufficient rejection at notch frequency and the out of band interference signals are not yet eliminated. Notch depth of these low-Q filters can be improved by increasing the order of the filter. However, this approach has a negative impact on the pass-band insertion loss which degrades the UWB receiver sensitivity. In order to design resonators with high quality factor, many solutions exist in the literature. Firstly, lossy resonators can use negative resistance circuits which aim to compensate for the overall losses of the filter at the working frequency. However, this very high quality factor resonator is obtained at the expense of degradation in circuit linearity and power consumption. Secondly, pre-distortion techniques can improve significantly the resonator quality factor [5], [6]. However, this improvement is obtained at the expense of increasing the pass-band insertion losses. Thirdly, the stopband performances of the filter can be improved by using the approach of phase cancellation between two or more signal paths [7]. By this way, a high stop-band attenuation level can be obtained at the expense of filter size.

The initial objective of the project was to design RF components such as UWB antenna and notch filter on flexible substrate. The UWB receiver which is designed using CMOS $0.13 \mu \mathrm{m}$ technology will be reported on a grounded area of the flexible substrate and connected to the output of the notch filter using wire-bonding. However, because of the time required for the electrical characterization of the flexible substrate, one choose to design the notch filter using known substrate to test first its performances and to perform the characterization of the flexible substrate at the same time. The performances of the UWB antenna have already been tested using Rogers 4003 substrate [8].

In this paper, the electrical characterization of the flexible substrate has been performed by using a microstrip ring 
resonator. Also, the design of a compact passive notch filter using the approach of phase cancellation is reported. This filter uses two parallel branches that support signal flow in the forward direction, with one branch assigned a band-pass response designated at notch frequency, and the other an allpass transmission line segment designed to produce a $180^{\circ}$ of phase difference at the notch frequency. The filter is fully simulated using HFSS Ansoft simulation tools.

\section{HIGH FREQUENCY CHARACTERIZATION OF FLEXIBLE SUBSTRATE}

To determinate the electrical properties at high frequencies of a flexible substrate, many techniques exist in the literature. However, the most used methods are based on resonator methods [9]. Indeed, this technique consists of designing a resonator at frequency f0 while using approximate values of flexible substrate electrical parameters $(\mathrm{Er}, \tan \delta)$. Once the measurements are done, the frequency shift of the resonator and the losses introduced at the central frequency correspond to the inaccuracy of the parameters considered in the simulation. A sensibility analysis of the filter response is then made on the the electrical parameter values $(\mathrm{Er}, \tan \delta)$ to fit with measurement results.

The resonator considered in this paper is that of a ring resonator shown in Fig. 1. The center frequency depends on the dielectric constant of the substrate as well as the dimension of the ring [9]. Using the equations reported in [9], four resonators are designed at four different frequencies of the UWB bandwidth $(3 \mathrm{GHz}, 5 \mathrm{GHz}, 7 \mathrm{GHz}$ and $9 \mathrm{GHz}$ ) in order to obtain the substrate electrical properties in the required band. The circuits are designed using flexible Kapton-based substrate with a thickness of $125 \mu \mathrm{m}$. This substrate was used because of its high temperature resistance during the wire-bonding process [10]. The electrical characteristics of the substrate used to simulate the rings were set to $\mathrm{Er}=3.5$ and $\tan \delta=0.0026$. The gaps (s) between the feeding lines and the ring are set to the minimum distance $(S=150 \mu \mathrm{m})$ allowed by the design rules to maximize the coupling coefficient.

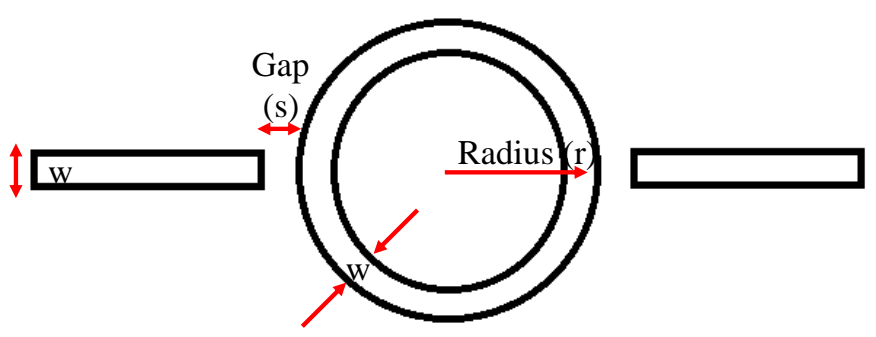

Fig. 1. Microstrip ring resonator configuration diagram.

Our measurements are performed using Rohde \& Schwarz Vector Network Analyzer (VNA). A through-reflect-line (TRL) calibration is planned in order to cancel the effects of the input and output feeding lines. The fabricated ring resonators are presented in Fig. 2. Measured S21 magnitude vs. frequency data were then inserted in an Excel sheet and the dielectric constant and loss tangent were extracted based on formulas of [9] (see Table I). Plots of measured S21 with respect to the frequency for three ring resonators are presented in Fig. 3. Using the extracted dielectric constant and loss tangent, the three ring resonators are re-simulated and a good correlation between measurement and simulation is observed as shown in Table II.

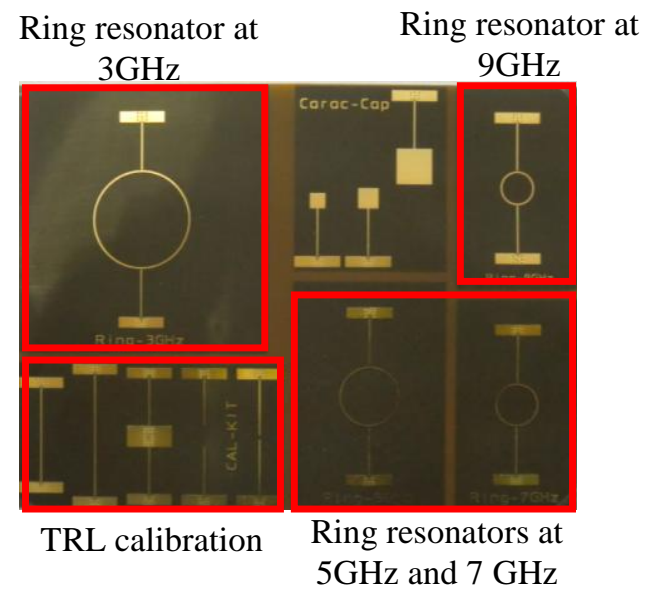

Fig. 2. Fabricated ring resonators on $125 \mu \mathrm{m}$ Kapton-based substrate.

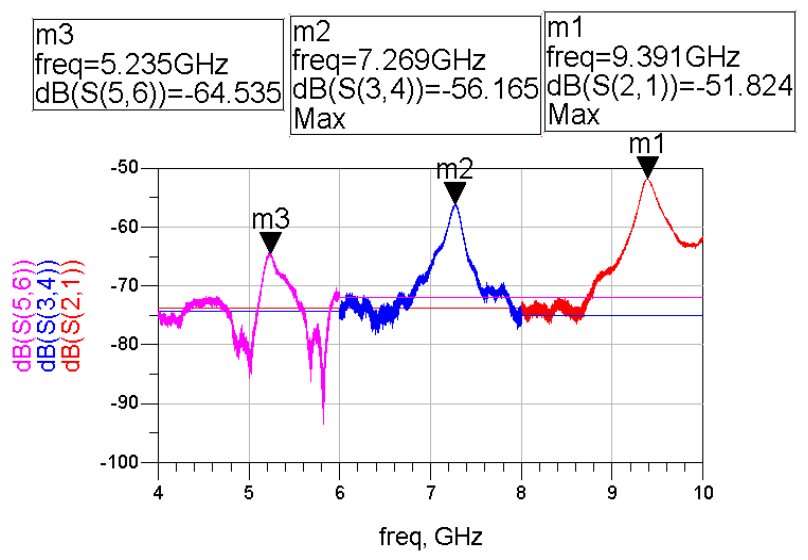

Fig. 3. S21 vs. frequency for three ring resonators.

TABLE I. EXCTRACTION OF DIELECTRIC CONSTANT AND LOSS TANGENT FROM RING RESONATOR MEASURMENT

\begin{tabular}{|l|c|c|c|c|}
\hline $\begin{array}{c}\text { Ring } \\
\text { frequency }\end{array}$ & $\begin{array}{c}\text { Measurement } \\
(\text { freq })\end{array}$ & $\begin{array}{c}\text { Measurement } \\
(\boldsymbol{d B} \mid \boldsymbol{S} 21)\end{array}$ & $\boldsymbol{E r}$ & tan $\boldsymbol{D}$ \\
\hline Ring at $9 \mathrm{GHz}$ & $9.391 \mathrm{GHz}$ & -51.824 & 3.29 & 0.0021 \\
\hline Ring at $7 \mathrm{GHz}$ & $7.269 \mathrm{GHz}$ & -56.165 & 3.27 & 0.0022 \\
\hline Ring at $5 \mathrm{GHz}$ & $5.235 \mathrm{GHz}$ & -64.535 & 3.27 & 0.0023 \\
\hline
\end{tabular}

TABLE II. RING RESONATORS SIMULATED AND MEASURED RESULTS

\begin{tabular}{|l|c|c|c|c|}
\hline $\begin{array}{c}\text { Ring } \\
\text { frequency }\end{array}$ & $\begin{array}{c}\text { Simulation } \\
(\text { freq })\end{array}$ & $\begin{array}{c}\text { Measurement } \\
(\text { freq })\end{array}$ & $\begin{array}{c}\text { Simulation } \\
(\boldsymbol{d B} \mid \boldsymbol{S} 21)\end{array}$ & $\begin{array}{c}\text { Measurement } \\
(\boldsymbol{d B} \mid \boldsymbol{S} 21)\end{array}$ \\
\hline $\begin{array}{l}\text { Ring at } 9 \\
\mathrm{GHz}\end{array}$ & $9.39 \mathrm{GHz}$ & $9.391 \mathrm{GHz}$ & -48.6 & -51.824 \\
\hline $\begin{array}{l}\mathrm{Ring} \text { at } 7 \\
\mathrm{GHz}\end{array}$ & $7.21 \mathrm{GHz}$ & $7.269 \mathrm{GHz}$ & -51.4 & -56.165 \\
\hline $\begin{array}{l}\mathrm{Ring} \text { at } 5 \\
\mathrm{GHz}\end{array}$ & $5.22 \mathrm{GHz}$ & $5.235 \mathrm{GHz}$ & -59.78 & -64.535 \\
\hline
\end{tabular}

III. DESIGN OF HIGHLY SELECTIVE CHANNELIZED FILTER

Once the electrical properties of the flexible substrate are extracted, it is henceforth possible to design RF components 
such as the UWB antenna and notch filter. However, in this paper, the notch filter is designed using another substrate for the reasons given in the introduction. The concept of the passive two-path notch filter is presented in Fig. 4. A portion of the input signal is coupled to the output of the filter through the band-pass filter which is designed at the notch frequency, while the remaining signal travels to the output through an all-pass segment line. If at a certain frequency, the two portions of the signal reach the output of the filter with the same amplitude but with a $180^{\circ}$ of phase difference between each other, then the signal at this frequency is canceled and a theoretical infinite attenuation is obtained.
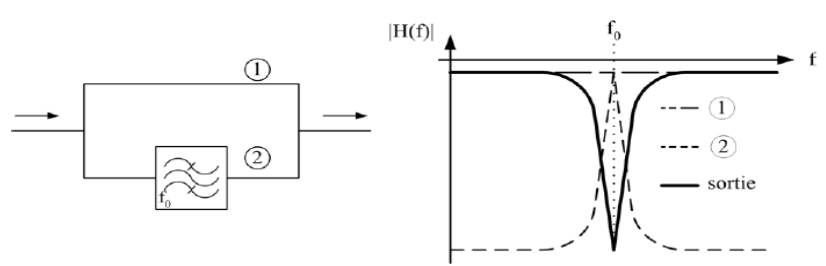

Fig. 4. Two-path notch filter concept.

Based on the technique described above, a notch filter is designed with a central frequency of $2.4 \mathrm{GHz}$. A multilayer $406 \mu \mathrm{m}$ thick Rogers 4003 substrate having a $35 \mu \mathrm{m}$ cooper metallization was used for the design. The band-pass resonator of characteristic impedance $\mathrm{Z} 0$ is tuned at $\mathrm{f} 0$ and the $50 \Omega$ delay line is designed to be a half-wavelength long at f0. In order to compact the structure, the band-pass filter was folded and the delay line was designed on the bottom layer of the substrate. This delay line is connected to the input and the output of the filter by metalized via holes as shown in Fig. 5 and Fig. 6. However, the size reduction of the filter was achieved at the expense of the filter rejection. Indeed, by folding the band-pass filter, the insertion loss at fo was increased due to the discontinuity of the lines which reduce the coupling factor. To overcome this problem, a short-circuited quarter-wavelength resonator line is added in parallel to the output of the filter to allow a better rejection of the filter (Fig. 5).

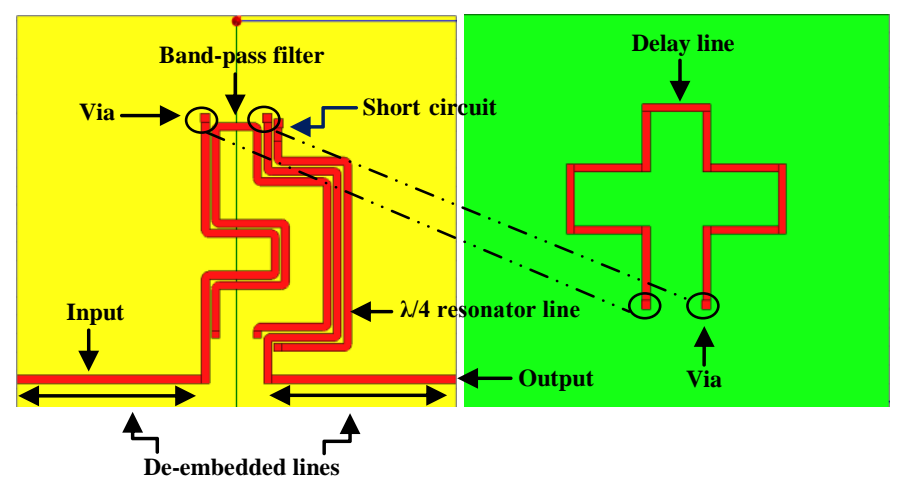

Fig. 5. Two-paths notch filter concept.

The filter is fully simulated using HFSS simulation tools. As depicted in Fig. 7, the filter features the following performances: The $3-\mathrm{dB}$ bandwidth is of $\sim 150 \mathrm{MHz}$, with a notch depth of around $20 \mathrm{~dB}$; the insertion loss is lower than $0.7 \mathrm{~dB}$ far from the notch frequency, and is of $0.4 \mathrm{~dB}$ in the
UWB bandwidth. The filter is also well matched with a return loss lower that $-10 \mathrm{~dB}$ beyond $\sim \mathrm{F} 0 \pm 200 \mathrm{MHz}$.

To simulate the complete structure, the integrated circuit (IC) chip is reported on a grounded area of filter and connected to the output of this later using wire-bonding as shown in Fig. 8. The Complete structure is then fully simulated and a plot of S21 with respect to the frequency is presented in Fig. 9. As can be noticed from this figure, the notch depth is slightly degraded. This behavior is due to the impedance mismatches and signal reflections at the carrier to chip transition.

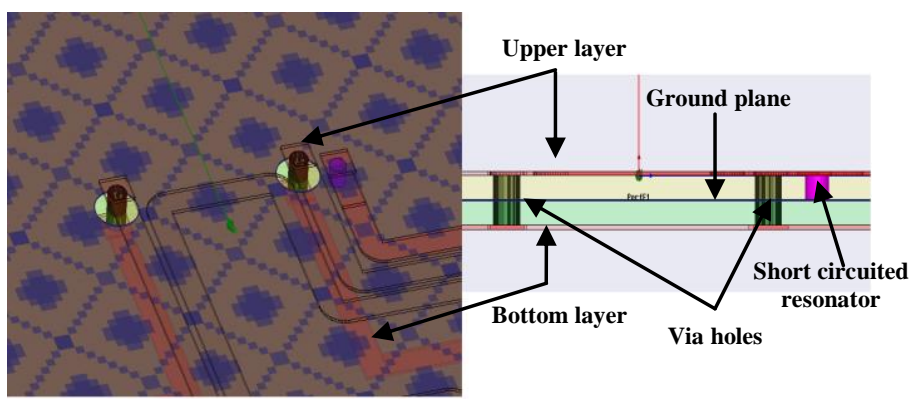

Fig. 6. Cross-view of the compacted two-path notch filter.

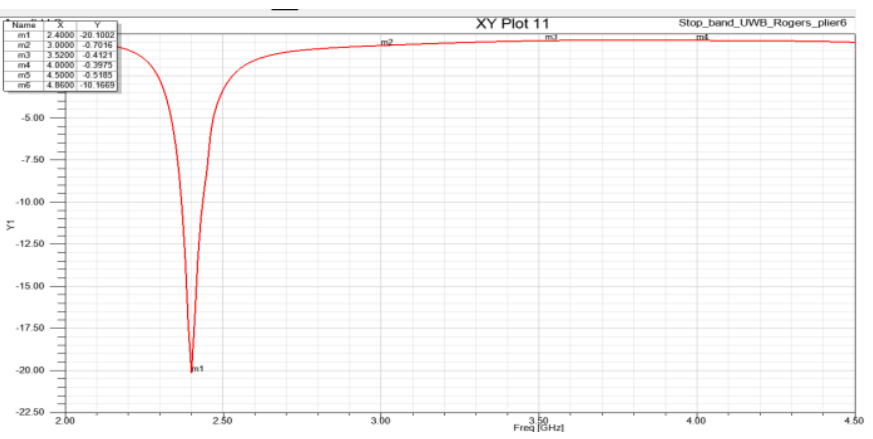

Fig. 7. Simulated S21 of the filter VS frequency.

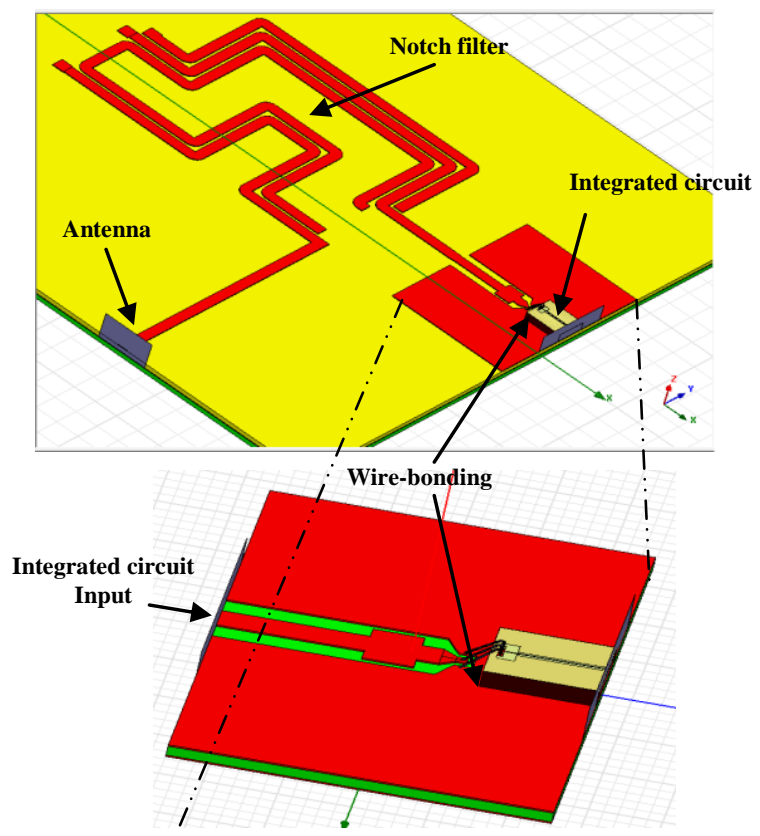

Fig. 8. Two-paths notch filter connected to the IC chip using wire-bonding. 


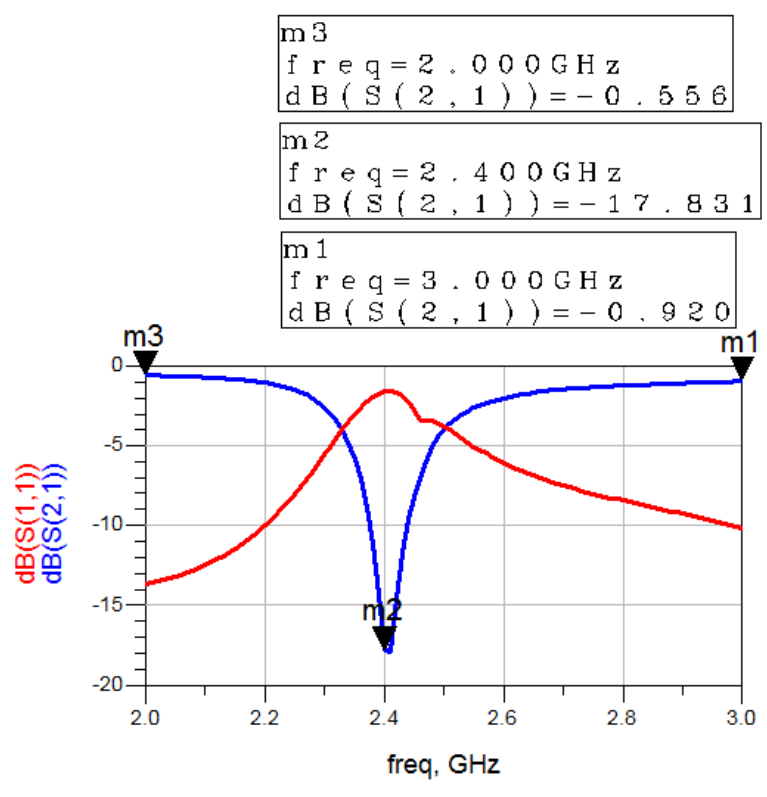

Fig. 9. Simulated S21 of the complete structure VS frequency.

The two-path notch filter is under realization (Fig. 10) and the measurement results will be compared to the simulation and shown in the final version of this paper.

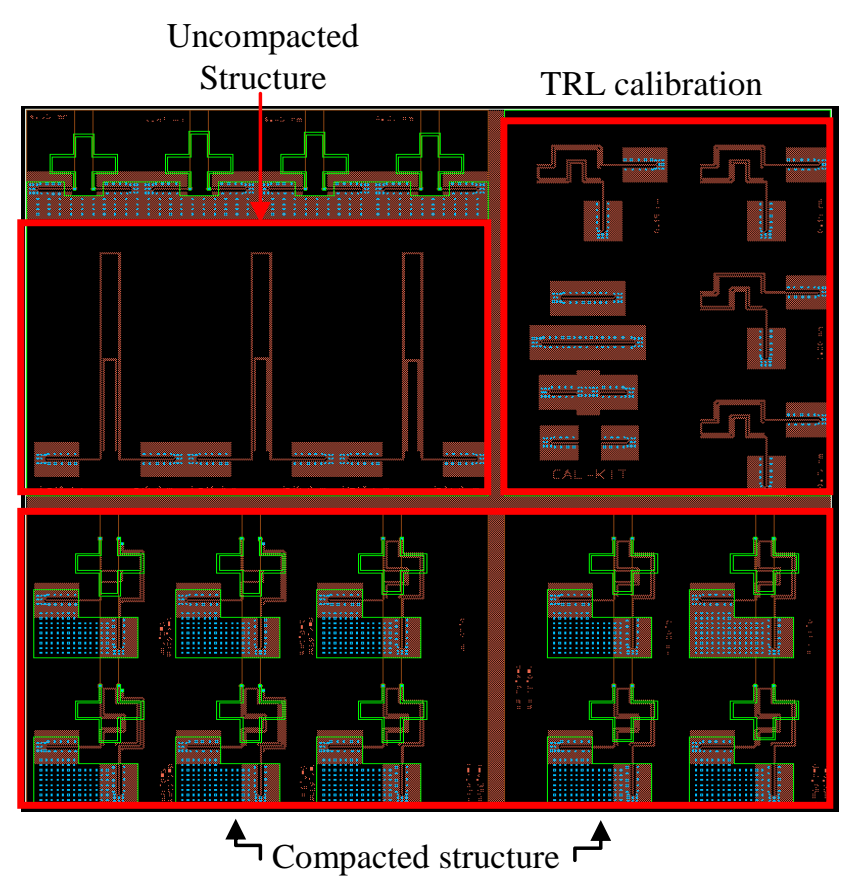

Fig. 10. Layout of the two-path notch filter.

\section{CONCLUSION}

In this paper, the dielectric characteristics of flexible Kapton-based substrate were investigated in the UWB range. In parallel with this task, a compact two-path notch filter was designed using multilayer Rogers4003-based substrate to first test its performances. This filter aims to protect the UWB receiver against interference signals coming from adjacent jammers. The notch filter was fully simulated using HFSS design tools, featuring high rejection at the central frequency and maintains low insertion loss $(0.7 \mathrm{~dB})$ far from the notch frequency. Currently, the filter is under realization, the measurement results will be presented in the final version of this paper. After this first step of test, it is intended to design the notch filter on flexible kapton-based substrate using the extracted dielectric characteristics done in this paper.

\section{REFERENCES}

[1] C. Rauscher, "Varactor-tuned active notch filter with low passband noise and signal distortion," ZEEE Truns. Microwave Z3eory Tech., vol. 49, no. 8, pp. 1431-1437, Aug. 200 1.J. Clerk Maxwell, A Treatise on Electricity and Magnetism, 3rd ed., vol. 2. Oxford: Clarendon, 1892, pp.68-73.

[2] J. D. Rhodes \& I. C. Hunter, "Synthesis of reflectiom-mode prototype networks with dissipative circuit elements," IEE Proc.-Microw. Antennas Propag., vol. 144, no. 6, pp. 437- 442, Dec. 1997.

[3] M. Berggren, T. Kugler, T. Remonen, D. Nilsson, M. Chen, P. Norberg, "Paper electronics and electronic paper," Proc. of the 2001 IEEE Conference on Polymers and Adhesives in Microelectronics and Photonics, pp. 300-303, October 2001.

[4] S. Basa, A. Rida, M.M. Tentzeris, "Design and development of novel miniaturized UHF RFID tags on ultra-low-cost paper-based substrates", Microwave Conference, 2006. APMC 2006. Asia-Pacific, Dec. 2006

[5] M. Yu, R. Cameron, D. Smith, V. Dokas and Y. Wang, "Symmetrical Realization for Predistorted Microwave Filters", IEEE MTT-S International Microwave Symposium Digest, 2005

[6] M. Yu, W-C. Tang, A. Malarky, V. Dokas, R. Cameron, Y. Wang, "Predistortion Technique for Cross-Coupled Filters and Its Application to Satellite Communication Systems", IEEE TRANSACTIONS ON MICROWAVE THEORY AND TECHNIQUES, VOL. 51, NO. 12, DECEMBER 20032505.

[7] D-R. Jachowski, "Passive enhancement of resonator Q in microwave notch filters", IEEE MTT-S International Microwave Symposium Digest, 2004

[8] A. Chami, O. Fourquin, G. Clementi, J.-Y. Dauvignac, N. Fortino, S. Bourdel, J. Gaubert, P. Brachat, G. Kossiavas, "Design and integration of UWB antennas for high data rate miniature impulse radio transmitter", Proceedings of the 5th European Conference on Antennas and Propagation (EUCAP), April 2011

[9] L. Yang, A. Rida, R. Vyas, "RFID Tag and RF Structures on a Paper Substrate using Inkjet-Printing Technology", IEEE Transactions on Microwave Theory and Techniques, Dec. 2007

[10] DuPont ${ }^{\mathrm{TM}}$ Kapton ${ }^{\circledR}$ HN , Technical Data sheet, http://www2.dupont.com/Kapton/en_US/assets/downloads/pdf/HN data sheet.pdf. 